\title{
30 YEARS OF TRANSDISCIPLINARY RESEARCH: A LETTER TO SPRINGER
}

\author{
Vilmos Katona
}

Faculty of Engineering and Information Technology, University of Pécs, 2 Boszorkány utca, Pécs 7624, Hungary

E-mail: katwilat@gmail.com, https://medbiotech.academia.edu/VilmosKatonaPhD

ORCID: 0000-0002-0299-2897

Dear Editor,

My name is Vilmos Katona, the new editor-in-chief of Symmetry: Culture and Science journal, and I would like to propose a book for publication by Springer.

The occasion of this proposal is that Gyuri Darvas, our former editor-in-chief and founder of the journal resigned after 30 years of work, and the community of the International Symmetry Association wants to commemorate him with an honorary book. We plan to publish a 224 to 256 pages book that would include the best articles of the journal's past 30 years, possibly including that of Benoit Mandelbrot or Nobel-laureates like Dan Shechtman, Gerard ‘t Hooft or Steven Weinberg.

Because our journal is interdisciplinary from the start, it has not just mathematical, but historical, geological, educational and architectural thematic issues-any fields that had to do with the science of symmetry. We have already started a conversation with Sarah Annette Goob, who understands the benefits of this irregular nexus between the seemingly distinct terrains of science and humanities. Our community is glad at finding 
an opportunity to involve our book in a series indexed by Scopus, for example the Trends in the History of Science.

The book would contain articles published before in the journal Symmetry. Due to the limited copies and print-only version, the older articles are neither available online, nor in book stores anymore. Our customers has no open access to newer papers, one may only download them for a certain price. Symmetrion, the publisher of Symmetry, is the only owner of the copyright of these materials, and will grant Springer the right to republish them. In case of additional photographs, we will contact the owners and provide Springer with their copyright transfer.

Since Symmetry is a refereed journal from the start, all the articles had been reviewed by professionals. We can send you samples of the filled review forms in case Springer would require them. You may also find more about our aims and scope, ethics, and authors' instruction, the latter of which contains a detailed description of our peer-review process. Articles may range from the 1990's until nowadays, but the book would reorganize them in a thematic, less chronological, order, restructuring each article into the form of chapters with the addition of necessary comments and updates. An extended introduction would summarize the purpose of the book. More importantly, it would introduce the selected articles not just as documents from the history of science, but as actualized materials enhancing contemporary researches.

The book could address the community of the International Symmetry Association: its funding supporters, authors and reviewers, together with scientists, artists, and institutes from all over the globe. Beyond that, it could be more than interesting to those who regard symmetry an individual discipline that attracts arts, philosophy and science in their various specific fields. Our proposed book aims at conveying to them knowledge, methods, and novelties which are applicable to their main fields of study and creative work. We are able to send you the manuscript within a reasonable time. Symmetry's editorial team hopes that we can cooperate on this matter for our mutual benefit.

Best wishes,

Vilmos Katona Ph.D.

Editor-in-Chief, Symmetry: Culture and Science 


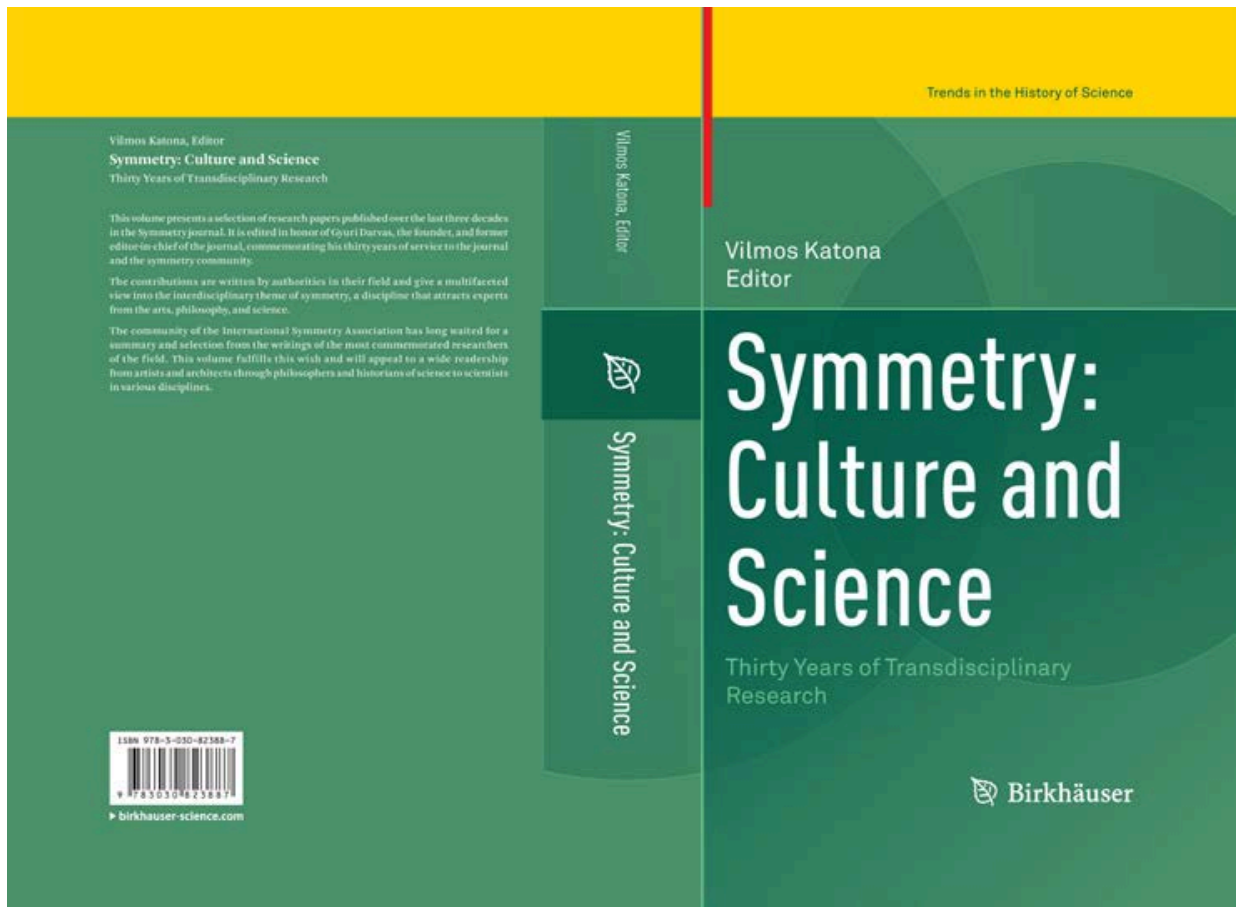

Vilmos Katona, Editor

Symmetry: Culture and Science: Thirty Years of Transdisciplinary Research ISBN 978-3-030-82388-7

Information and product flyer: https://www.springer.com/de/book/9783030823887

This volume presents a selection of research papers published over the last three decades in the Symmetry journal. It is edited in honor of Gyuri Darvas, the founder, and former editor-in-chief of the journal, commemorating his thirty years of service to the journal and the symmetry community. The contributions are written by authorities in their field and give a multifaceted view into the interdisciplinary theme of symmetry, a discipline that attracts experts from the arts, philosophy, and science.

The community of the International Symmetry Association has long waited for a summary and selection from the writings of the most commemorated researchers of the field. This volume fulfills this wish and will appeal to a wide readership from artists and architects through philosophers and historians of science to scientists in various disciplines. 